\title{
Utilizing Correlated Light and Electron Microscopy to Examine Alpha-Synuclein Pathology in a Mouse Model of Parkinson's Disease
}

\author{
Allison Schaser ${ }^{1,2 *}$, Claudia S. López ${ }^{3,4}$ and Vivek Unni ${ }^{1,2}$ \\ 1. Jungers Center for Neuroscience Research, Oregon Health \& Science University, Portland, OR, USA. \\ 2. Department of Neurology, Oregon Health \& Science University, Portland, OR, USA. \\ 3. Department of Biomedical Engineering, Oregon Health \& Science University, Portland, OR, USA. \\ 4. Multiscale Microscopy Core, Oregon Health \& Science University, Portland, OR, USA. \\ * Corresponding author: schaser@ohsu.edu
}

Parkinson's disease (PD) is a progressive neurodegenerative disorder for which there are currently no treatments to slow, halt, or reverse the disease process. The disease is defined by the accumulation of the protein alpha-synuclein into aggregates known as Lewy inclusions, but how these aggregates initiate and propagate to various locations throughout the brain is unknown [1]. To develop targeted disease modifying therapies, it is important to understand how aggregated forms of alpha-synuclein are transported throughout the nervous system to rationally intervene to stop and/or reverse the process. The goal of this study was to use correlated light and electron microscopy (CLEM) imaging approaches to determine both the structure and location of alpha-synuclein pathology in a mouse model of PD [2].

Previous work suggests that alpha-synuclein pathology may, in part, propagate when fibril aggregates of alpha-synuclein spread from cell-to-cell [3]. Alpha-synuclein pathology propagation can be induced by the exogenous application of small, in vitro-generated alpha-synuclein pre-formed fibrils (PFFs). Previous research has also shown that the application of PFFs causes aggregation of endogenous alphasynuclein and resultant pathology first at the site of injection and then in connected brain regions in a time dependent manner [4-5]. The specificity and inducibility of the PFF approach makes it an ideal system to examine the structure and location of alpha-synuclein aggregate pathology. All experiments in this study were carried out using the PFF approach in an alpha-synuclein transgenic mouse model developed in the Unni laboratory. This mouse model overexpresses the aggressive disease-associated human A53T mutation via the mouse prion promotor and is tagged with a green fluorescent protein (Syn-GFP). This mutation leads to more rapid development of alpha-synuclein pathology. At the light level, alpha-synuclein pathology in this model was defined by the aggregated nature and morphology of the fluorescent signal of the Syn-GFP. Syn-GFP pathology has been previously validated through fluorescent recovery after photobleaching and fixed tissue immunohistochemistry techniques [5].

Brain tissue from Syn-GFP mice was examined in both control (un-injected) and experimental (PFFinjected) animals. Specifically, in the experimental group, $2.5 \mu \mathrm{L}(2 \mathrm{mg} / \mathrm{mL})$ of PFFs was injected into a single site in the motor cortex (AP -1.5, ML -1.0, DV $-0.3 \mathrm{~mm}$ from skull). Pathology was then allowed to develop for 1 month. At the 1 month post-injection time point, mice were euthanized and their brains were fixed and sliced for CLEM analysis. Un-injected littermates were used as controls. Tissue was prepared for CLEM experiments using methods developed in collaboration with the Oregon Health \& Science University (OHSU) Multiscale Microscopy Core (MMC) and imaged using the MMC's FEI CorrSight $^{\mathrm{TM}}$ and Helios Nanolab ${ }^{\mathrm{TM}} 660$ microscopes. CLEM Images were analyzed using FEI MAPS ${ }^{\mathrm{TM}}$ software packages [6]. 
CLEM techniques allowed for narrowing in on a field of interest specific to areas with Syn-GFP pathology, while still allowing for high quality EM images that provided the resolution necessary to examine the specific cellular structure that contained pathology within in the tissue (Figure 1). Using CLEM to narrow in on tissue of interest, as compared to traditional EM techniques, was of particular importance because Syn-GFP pathology is not ubiquitous throughout the tissue in this model. Results show the first direct evidence that Syn-GFP inclusions are fibrillar in nature and are present in neuritic structures following PFF-injection in this mouse model (Figure 1).

Future work will focus on continued improvement of the CLEM protocol to optimize tissue preservation and structural integrity within mouse brain samples. The goal of future work will be to elucidate possible mechanisms of transport to target perturbation of these mechanisms to halt or reverse the accumulation of abnormal alpha-synuclein that leads to progressive clinical disability in PD [7].

\section{References:}

[1] M Spillantini et al., Nature 388 (1997), p. 839.

[2] P de Boer, J Hoogenboom and B Giepmans, Nat Methods 12 (2015), p. 503.

[3] K Luk et al., Proc Natl Acad Sci 196 (2009), p. 20051.

[4] A Sacino et al., Proc Natl Acad Sci 111 (2014), p. 10732.

[5] V Osterberg et al., Cell Rep 10 (2015), p. 1252.

[6] OHSU Multiscale Microscopy Core, https://www.ohsu.edu/xd/research/research-cores/multi-scalemicroscopy-core/ (accessed Feb 22, 2019).

[7] The authors acknowledge funding from the OHSU SOM Research Core Initiative Grant (Schaser) and the NIH P30 NS061800 (Aicher).
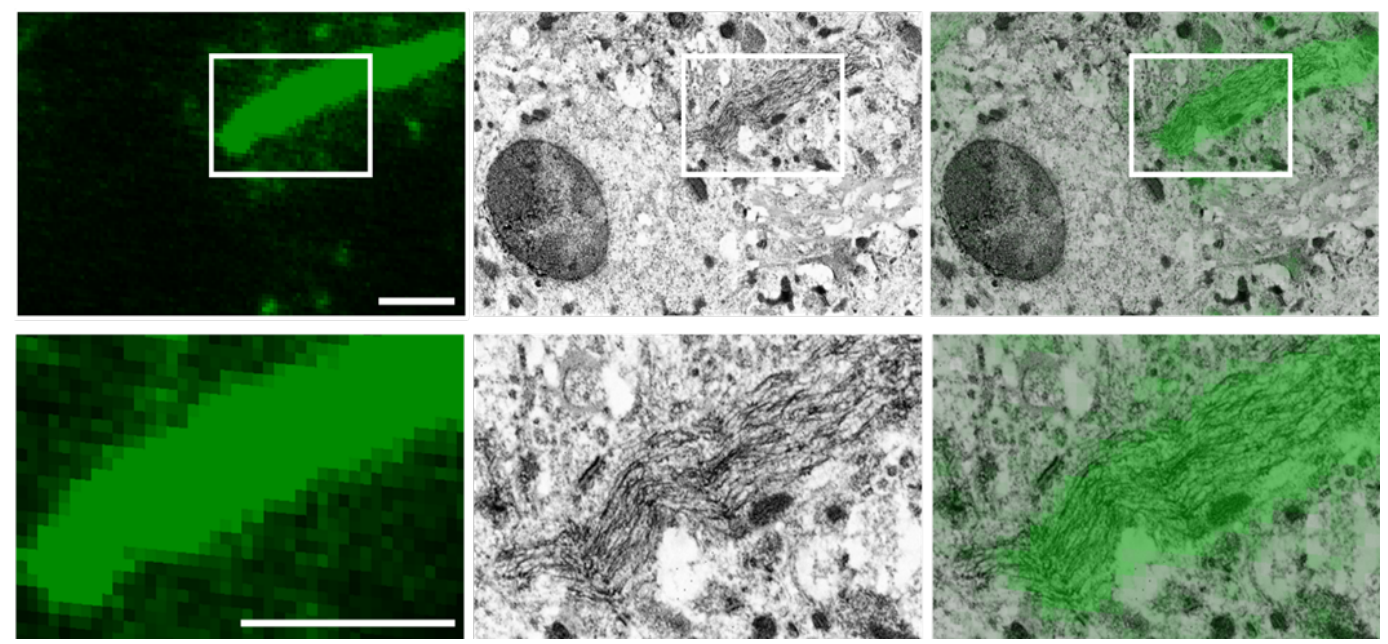

Figure 1. Representative CLEM Images. All images taken from the same section of PFF-injected SynGFP mouse brain tissue. Section was processed and embedded according to CLEM protocol, thin sectioned, and mounted for consecutive imaging sessions. Left Panel: Fluorescent images taken on the CorrSight fluorescent microscope show Syn-GFP staining in unknown structures. Middle Panel: Electron micrographs taken on the Helios scanning electron microscope in backscatter mode using MAPS $^{\mathrm{TM}}$ software to target the exact area of tissue imaged using the CorrSight in left panel. Right Panel: Overlays of both images show punctate Syn-GFP staining localized to terminals and aggregated Syn-GFP localized to fibril structures in a neuritic process. Bottom row shows zoomed in images at location noted by square in top row.

Scale bar $=2 \mu \mathrm{m}$ 